\title{
The Need to Know Algebra Skills, Misconceptions, Misapplications and Weaknesses of Students
}

\author{
Cristina Eccius-Wellmann \\ Universidad Panamericana, Guadalajara, Mexico
}

\begin{abstract}
Misapplications, misconceptions, and weaknesses in algebra hinder students to understand other topics like finance, operations, microeconomics and other branches of business schools. To help students effectively to learn it is necessary to know what are the most worrying arithmetic and algebraic errors they show and which misconceptions they might underlie. A 42-item test was administrated to 270 freshmen who enter Universidad Panamericana, “Escuela de Ciencias Económicas y Empresariales”, to investigate their arithmetic and algebraic skills, misconceptions, misapplications, and weaknesses, and it was found that a vast majority had a low performance. Marking an item as incorrect would not help students to overcome their difficulties. Five items, with the most worrying arithmetic and algebraic misunderstandings were analyzed via students' processes to know the possible causes of the arithmetic and algebraic errors in order to apply effective strategies to remediating these difficulties. students' procedures often show very clear about their thinking and their misconceptions. Several different misconceptions in one and the same item were found very often. Therefore, it is imperative to analyze every student's answer to know which might be the reason of the error. Every misconception has to be treated in different ways. It was established, that students show basic arithmetic and algebraic errors, like order of operations, conjoin problems, improper simplification strategies, and other problems. Students who enter Universidad Panamericana came from many different high schools in the west and north-west of Mexico and the transmission of these results have the intention of highlighting the importance of detecting misconceptions when they appear in school mathematic lessons, in spite of having less mathematic problems in college.
\end{abstract}

Keywords: systematic error, algebraic expressions, simplifying algebraic expressions, bracket expansion

\section{Introduction}

Algebra is an important topic. The applications of algebra can be found in almost all branches of science, and in administration and finance. Many college students encounter serious difficulties in solving linear equations, quadratic equations, and in simplifying elementary algebraic expressions. They also exhibit several errors in basic arithmetic operations, where their understanding of the number sense is central. How can they comprehend finance, administration, and microeconomics, if they do not have a minimum of algebraic understanding? For example, the performance of a compound interest, like: $M=C\left(1+\frac{0.03}{12}\right)^{15}$ may be difficult for students. Many of these students do not finish their studies, because they are not able to overcome their

Cristina Eccius-Wellmann, Dr., Ph.D., Business school, Universidad Panamericana.

Correspondence concerning this article should be addressed to Cristina Eccius-Wellmann, Universidad Panamericana, campus Guadalajara; Prol. Calz. Circ. Pte. \# 49; Zapopan, Jalisco. C.P. 44010, Mexico. E-mail: ceccius@up.edu.mx. 
algebraic problems. Therefore, it is necessary to identify, as quickly as possible, the arithmetic and algebraic skills which students are bringing to college from the earlier studies in school. Due to the situation that students of Universidad Panamericana come from different high-schools in Mexico, it is important to know what skills, misconceptions, and weaknesses students have for a further and better understanding of teaching.

\section{Investigation Questions}

(1) Which are the most worrying algebraic misconceptions students bring from high school?

(2) Which misconceptions and students' thinking might underlie every procedure in spite of looking for effective help for students?

This paper shows the performance of 270 students in a diagnostic test, making evident not only the low percentage of correct answers. It highlights the most worrying misconception, misapplications, and weaknesses students showed. This paper does not pretend to be exhaustive, it will analyze some student's procedures that clearly manifest their difficulties. The designed test applied to the students consisted in 42 items and the most common errors, in five selected items, were found in arithmetic operations, including the order of operations, simplifying algebraic expressions, solving linear and quadratic equations, graphing a linear function, topics of brackets expansion, algebraic fractions, and factorizing an algebraic expression.

\section{Theoretical Framework}

Research into errors provides a rich source of knowledge about the processes and mechanism used by students to simplify and factorize algebraic expressions in solving equations and graphing linear functions. The identification and classification of students' errors help to know what they have not learned, and what they misslearned. It will help teachers and professors to focus on effective strategies for remediating arithmetic and algebraic difficulties and misconceptions. Researches on the development of algebraic understanding by identifying the errors committed by students are well documented (Vlassis, 2002; Warren, 2003). However, the persistence of these errors in college students is not often studied (Eccius, 2008; Liebenberg, 1997). Researches on errors in early arithmetic operations are also reported (Padberg, 1996, 2005; Radatz, 1979), but little attention is paid until now on errors in number sense of college students (Eccius, 2008). A few typical and systematic errors in arithmetic and algebra are discussed in the following sections.

Order of operations: The order of operations is crucial when students calculate arithmetic operations or transform algebraic expressions. Simple arithmetic operations make it able to analyze what conceptions students have about the order of operations (Eccius, 2008). The calculation of $25-5 \cdot 2+4=$ may show many different interpretations in the results. The most common interpretation is to work out the expression, from left to right, without observing the hierarchy of operations.

Conjoin errors: The expression "conjoin" means, in arithmetic and algebra, how letters and numbers are attached and the interpretation is given. Conjoin errors occur, when students do not understand the different meanings of conjoin in arithmetic and algebra. Arithmetic meaning of 53 is $50+3$ (an additive meaning); algebraic meaning of 3x is 3 multiplied by x (multiplicative meaning) (Nolte, 1991; Malle, 1993). Common conjoin errors are:

- $4+3 x=7 x$; in reducing algebraic expressions;

- $3+\mathrm{x}=3 \mathrm{x}$; the interpretation of $3 \mathrm{x}$, for some students, is the same as the additive result of $3+\mathrm{x}$, because 
they do not know the different "conjoin” meanings in arithmetic and algebra;

- This error occurs, also because students cannot accept open expressions, they think they are incomplete, and want to find a close expression (Malle, 1993; Nolte, 1991). Tirosh, Even, and Robinson (1998) referred to this thinking as "lack of closure".

It is easy to find further errors due to conjoin errors, for example, in an equation: $-3 x=9$ instead of dividing both sides of the equation by $-3,3$ was added to both sides. $x=12$. The interpretations may look like $-3 \mathrm{x}=9$, which is the same as $-3+\mathrm{x}=9$, so the adequate operation might be adding 3 to both sides.

The equal sign: In arithmetic operations, an equal sign represents the order of doing something. The interpretation of an equal sign changes in algebra, it may have not the connotation of an order. Students may incur in errors if they follow seeing the equal sign as an order to do something (Malle, 1993; Nolte, 1991; Tietze, Klika, \& Wolpers, 2000). Kieran (1979) investigated that many students tend to equal an algebraic expression to zero " $=0$ ", when they are asked to simplify or factorize an algebraic expression. Students try to find a final answer, something like " $x=5$ ", because they could not accept an " $x+5$ " as an answer. Students often use the equal sign in successive transformations and simplifications, and the left side of an equal sign does not have the same value of the right side (Eccius, 2008).

Simplification techniques and view of operation signs: The conceptions, when and how a fraction may be simplified, are poorly delimitated by students. Some specific exercises, like cross schemes, create over-generalizations of methods to simplify fractions. Students often have the conception that they can cross in a fraction, all "things" are equal in the numerator and denominator. Operation signs are often treated by students as factors ("things") (Eccius, 2008). For example, root signs which are simplified in an algebraic fraction expression. Another frequent error consists in simplifying a fraction, without understanding the fundamental principle of the fractions: a fraction may be simplified, dividing (the whole) numerator and the (whole) denominator by a quantity different of zero. So it is possible for students to simplify a fraction with an addition or a subtraction sign in the numerator or denominator.

Misconceptions with distribution rules: Problems with an overgeneralization of the distribution law are reported by Malle (1993), and they are also referred to linearization problems. Students may incur in errors, because they apply a distribution law to transformations where they are not correct, like:

$$
(a+b)^{2}=a^{2}+b^{2}
$$

Often these misconceptions have a deeper origin. Students work out schemata that are not sufficiently limited. For instance, the distribution works for:

$$
\frac{a+b}{c}=\frac{a}{c}+\frac{b}{c}
$$

but does not for:

$$
\frac{a b}{c}=\frac{a}{c} \times \frac{b}{c}
$$

the metacognition lies on the difference an operation sign.

There are many other errors in arithmetic and algebraic operations (Eccius, 2008), but they could not all be discussed in this paper.

Analyzed Items: It is also impossible to analyze the 42 items in this paper (Appendix). Items 1, 13, 17, 25, and 30 were chosen to look for the performance, errors, misconceptions, and weaknesses of the students: 


$$
\begin{aligned}
& \text { 1. } 25-5 \cdot 2+4=\quad \text { 13. } \frac{(x-5)^{m+2}}{(x-5)^{m}}=\quad \text { 17. } \frac{\sqrt[3]{z^{4}}}{\sqrt[3]{z}}= \\
& \text { 25. } 9 y^{2}+6 y+1=\quad 30 . \quad \frac{y(y+2)+(y+2)}{y+2}=
\end{aligned}
$$

In the next section, each of these chosen items will be discussed to analyze the purpose of the application.

\section{Research Design}

The test consists of 42 items of secondary school arithmetic and algebraic operations and simplifications.

The first part of the study is an overall counting of correct answers in the test. The study presents a graph of the obtained marks of 270 students. The percentage of correct answers is only a form to detect low performance in general. The important part of this study lies in the analysis of students' productions. Which misconceptions and students' thinking might underlie every procedure in spite of looking for effective help for students? For every item, the most common misconceptions and errors that are discussed in terms of student's thinking make inference of causes of the error. Some misconceptions are typical to systematic, others are very individual procedures. Not all misconceptions can be discussed in this paper.

Participants: 270 students who entered Business School at Universidad Panamericana, campus Guadalajara (2010), where they were tested.

Instrument: 42 items which are administrated to the students to investigate their difficulties and errors in understanding basic arithmetic operations and algebraic expressions, and in solving linear and quadratic equations (Appendix).

The quantification of the percentage of correct answers is a measure of the magnitude of the problem and justifies the need of attending these difficulties. The written procedures of the students give an insight in their thinking processes, their conceptions, misconceptions and weaknesses.

Item 1, Calculate: $25-5 \cdot 2+4=$

This item is referred to arithmetic operations and involves order of operations. Many different errors could be made by students. The most common error is to operate from left to right. Other errors occur, when students "see" brackets where there are none. Students with difficulties in arithmetic order of operations may show problems in algebraic performance, due to the view of operation hierarchy they have.

Item 13, Simplify: $\frac{(x-5)^{m+2}}{(x-5)^{m}}$

Order of operations is an important fact when students try to simplify or reduce this expression. Some students cannot distinguish a multiplication from an exponent, so they transfer multiplying operations to exponent operations. They have to apply exponent rules. If a student's view of this expression is primarily a fraction, the techniques used may cause error, because he/she may apply cross-schemes, not only with the basis expressions, but also with the exponents.

Item 17, Simplify: $\frac{\sqrt[3]{z^{4}}}{\sqrt[3]{z}}$

The main idea is to look for misconceptions like simplification of operation signs. Students cross out the root sign, in the numerator and denominator. This shows that students do not distinguish factors from operation signs.

Another possible error is a misunderstanding of the cubic root sign. 
Item 25, Factorize: $9 y^{2}+6 y+1=$

This item makes it possible to identify misconceptions in factorization. Some conjoin problems, and misinterpretations are in the identification of expressions and equations.

Item 30, Simplify: $\frac{y(y+2)+(y+2)}{y+2}$

The simplification of these algebraic fractions was designed to analyze the cross schemes errors. An easier algebraic expression (item 29) is often simplified correct by students, but in the next two items, students misuse cross schemes. They do not identify the structure of the expressions in the numerator and denominator. The addition sign in the numerator makes a direct simplification impossible, other techniques should be used.

It must be clear that in every item mentioned above, students may incur in different types of error, subsequently and also in non-typical errors. Analyzing the written procedure may show other errors than expected, but it is important to understand student's thinking.

Application of the test: Students answer the test without calculator. They have sufficient time and the test has no mark for them, it is only a form for knowing their abilities, procedures, misconceptions, and weaknesses.

\section{Results}

Part 1: Overall performance.

Figure 1 shows the performance of the 270 students in the test. The performance of the vast majority is low. Approximately $75 \%$ of the students did not reach $30 \%$ of correct answers in the test.

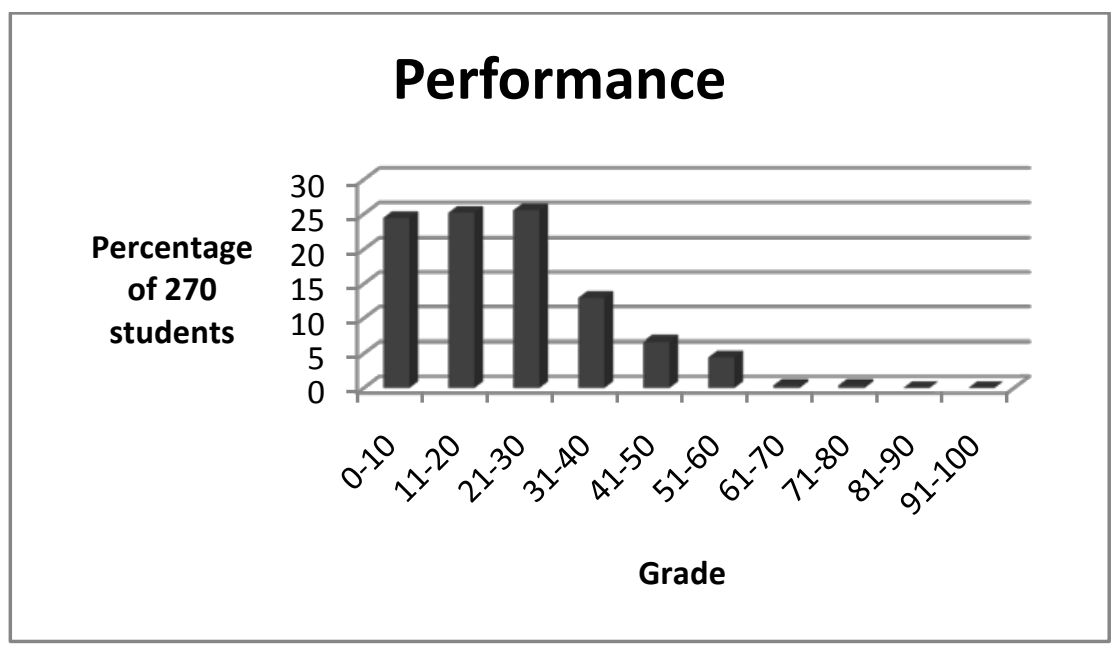

Figure 1. Performance of 270 students in 42 items.

Part 2: Percentage of correct answers in the chosen items (1, 13, 17, 25 and 30).

Figure 2 shows the percentage of correct answers in items 1, 13, 17, 25, and 30. Stating student's low performance in these five items would not help students. Only a deeper analysis of students' productions may help understand their thinking. The next section shows the common misconceptions of students, sometimes in a transcription, sometimes as scanned answers.

Students often mix various misunderstandings in one performance, so it might be difficult to state only one misconception in some students' performances. 


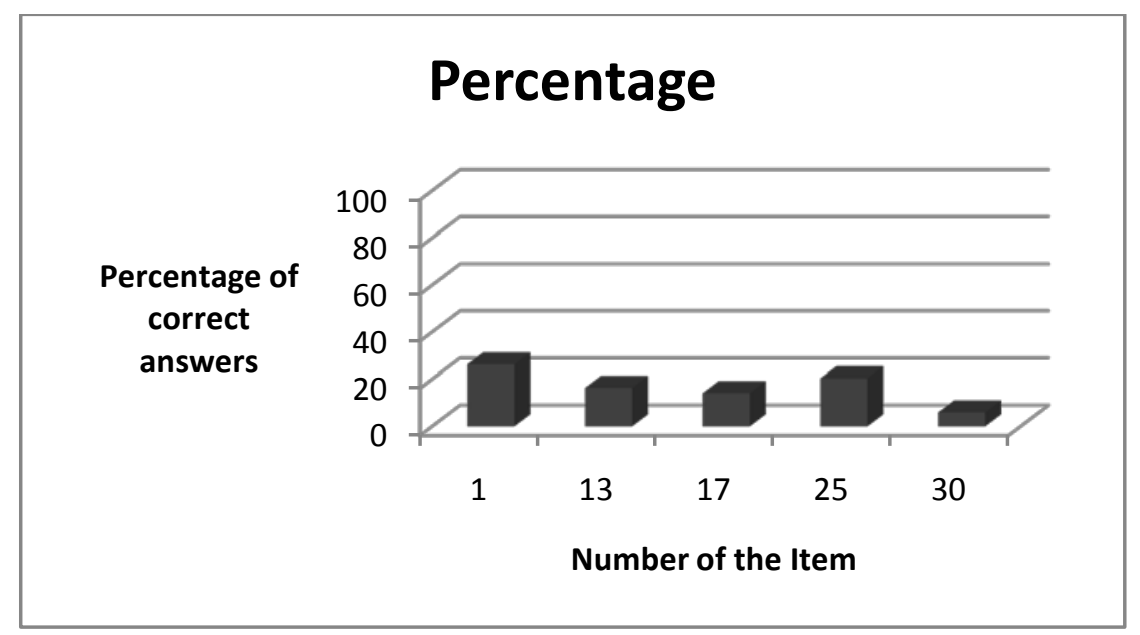

Figure 2. Percentage of correct answers in the five analyzed Items.

Part 3: Most common answers with error and the corresponding analysis.

Item 1, Calculate: $25-5 \cdot 2+4=$

(1) The most common error is the calculation from left to right.

$$
\begin{aligned}
& 25-5=20 \\
& 20 \times 2=40 \\
& 40+4=44
\end{aligned}
$$

This form of thinking may come from the mental calculus, which is trained in primary and secondary schools. When students affront the written form, they cannot distinguish the differences.

(2) Other errors are due to "set" brackets where they are not. This can occur in different ways.

$$
(25-5) \times(2+4)=20 \times 6=120
$$

(3) $25-(5 \times 2+4)=25-10-4=11$

Conclusions: When students have problems in the order of operations, they may have difficulties in a vast variety of arithmetic and algebraic operations.

Students often "set" or "imagine" brackets, which change completely the interpretation of the operation. Items with brackets are regularly well performed by students, but sometimes also misunderstood.

Item 13, Simplify: $\frac{(x-5)^{m+2}}{(x-5)^{m}}$

a.

$$
\frac{(x-5)^{m+2}}{(x-5)^{m}}=\frac{m+2}{m}=2
$$

$$
\frac{(x-5)^{m+2}}{(x-5)^{m}}=\frac{x^{m+2}-5^{m+2}}{x^{m}-5^{a}}
$$

c.

d.

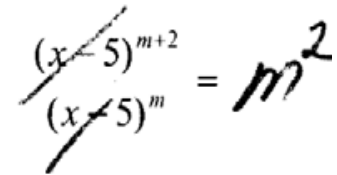

e.

$$
\frac{(x-5)^{m+2}}{(x-5)^{m}}=\frac{5 x^{2 m}}{5 x^{m}}=1 x^{3 n}
$$

(a) Both of the above performances are due to misconceptions of order of operations. Students do not identify that they have to consider first the exponents and then the division. If students look first on the fraction, they probably will perform cross-schemes. The first process show clearly the second error, a simplification of 
fractions, where the numerator has an addition-sign. Bases where lost, and character of the exponent lost too. Fraction is composed by the two exponents.

(b) The second performance probably misused cross schemes several consecutive times. In this performance, the base is not lost, it is 1 , but we cannot know, if the student applies a further correct exponent rule, or if he makes a cross scheme.

(c) The process can be seen as a linearization process. Students apply distribution rules to exponents. The student could not find a further process to simplify the expression.

(d) The process show a cross scheme. Afterwards, the student has the problem to state what the basis is and what should be the exponent. Due to the fact that sometimes students do not think of a cross scheme with result 1 , the bases are not existent.

(e) The students' process is evident for a conjoin problem. $(x-5)$ is written as $5 x, m+2$ is written as $2 m$. Further performance shows simplification techniques and a misunderstanding of exponent rules.

Conclusions: Students have to understand the structure of an algebraic expression. The structure may help them to correct misconceptions.

Item 17, Simplify: $\frac{\sqrt[3]{z^{4}}}{\sqrt[3]{z}}$

$$
\frac{\sqrt[3]{z^{4}}}{\sqrt[3]{z}}=z^{3}
$$

a.

(a) The most common error for this exercise is the interpretation of operations signs as "factors". The process is a clearly identification of the root signs as "things" that are equal in the numerator and denominator.

(b) Some misunderstandings in exponent rules with fractional exponents may show problems like this. Sometimes the rules that for teachers are obvious to make no sense for students.

Conclusions: For students, it is important to understand the underlying structure of the expressions and the signification of the rules. For understanding rules, students have to understand first what it means to have an exponent and how to interpret them.

Item 25, Factorize: $9 y^{2}+6 y+1=$

a.

$$
9 y^{2}+6 y+1=y(9 y+6)+1
$$

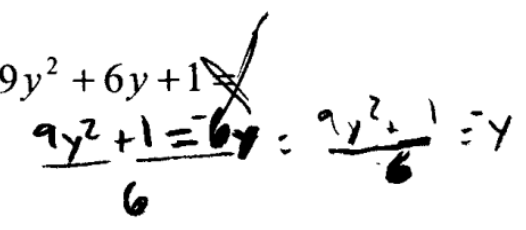

b.

$$
9 y^{2}+6 y+1=16 y^{3}
$$

$$
9 y^{2}+6 y+1=15 \gamma=\sqrt{1}
$$

d.

(a) Students' productions of trying to factorize this expression, sometimes failed, not in a mathematical wrong expression (there is no mathematical error in the answer), but in the understanding of it which is meant with a factorization.

(b) The factorization exercise makes some other misconceptions evident, like the conjoin problems. It can be found several different interpretations of conjoin difficulties (see d).

(c) The students' interpretation is a resolution of an equation. He/she establishes an inexistent equation: 
$9 y^{2}+6 y+1=0$ and makes further operations to solve the equation. The misconception is due to the fact that he/she tries to express the variable in terms of the same variable (a very common misconception, when students have to solve quadratic equations).

(d) An inappropriate use of the equal sign is exposed in the student's performance, and it also illustrates a conjoin problem. The probable thinking of the student: $9 y^{2}+6 y+1$ is the same as,

$15 y^{2}+1$, this is;

$15 y^{2}=1$; by rooting both sides

$15 y=\sqrt{1}$

Conclusions: Students show a variety of misconceptions in factorization exercises, it should be clear that the conception of factorization is misunderstood by students or it has no sense for them, because they do not try to factorize, they probably do not know what they are asked to do.

Item 30, Simplify: $\frac{y(y+2)+(y+2)}{y+2}=$

$$
\frac{y \cdot(y+2)+(y+2)}{y+2}=\mid y^{2}+2 y \quad \frac{y \cdot(y+2)+(y+2)}{y+2}=4
$$

a.

c.

$$
\begin{aligned}
& \frac{y \cdot(y+2)+(y+2)}{y+2}= \\
& y^{2}+3 y+z=y+2 \\
& y^{2}+2 y=0
\end{aligned}
$$

b.

d.

$$
\begin{gathered}
\frac{y \cdot(y+2)+(y+2)}{y+2}=\frac{y^{3}+4 y^{2}+4 y}{y+2} \\
\frac{\left.y^{2}+2 y\right)(y+2)}{y+2}=y^{3}+2 y^{2}+2 y^{2}+4
\end{gathered}
$$

$$
\begin{array}{ll}
\frac{y \cdot(y+2)+(y+2)}{y+2}= & \frac{(y)(2 y+4)}{y+2}
\end{array} \frac{y \cdot(y+2)+(y+2)}{y+2}=\frac{y}{y+2}
$$

(a) The shown performance above is the most common error. Students' misconceptions of simplifying this algebraic fraction correspond to cross schemes, without any limitations and metacognition. A further error is that students do not recognize the result of the simplification they made as a 1 . They omit the 1 , because they think of an elimination in the cross scheme.

(b) Cross scheme simplification make students' thinking about them as eradication evident. In this thinking, the result of the eradication is zero.

(c) The student identifies an equation in this algebraic expression. It is not clear if he/she states a 1 or a 0 at the right side of the equation. The performance of the multiplication and addition is correct. The equation is not solved.

(d) For this student, the brackets confuse the way of understanding the exercise. The addition sign is ignored. He/she understands the exercise as follows: 


$$
\frac{y(y+2)(y+2)}{y+2}=\frac{\left(y^{2}+2 y\right)(y+2)}{y+2}
$$

and does not simplify the expression, probably due to the fact that he wants to perform the multiplications.

(e) The student performs first the addition, without considering the order of operations. He/she does no further simplification.

(f) Two different misconceptions are in this process. The first one is a misconception in the distribution law: $a b / c$ is not equal to $(a / c)(b / c)$. The second error is eradication thinking in the cross scheme performance of the second fraction. The result of the simplification of the second fractions is zero for the student.

Conclusions: Students performances in simplifying an algebraic fraction show a diversity of algebraic misconceptions. Often students are not aware of what it means to have a factor in the numerator and denominator of the fraction. Cross schemes are often over-generalized.

\section{Conclusions}

The vast majority of the students entering Universidad Panamericana have a low performance in mathematics skills. Their achievement in algebra is hindered by common misconceptions and misunderstandings. They cannot concentrate their studies on different topics, because they fight with mathematics. This report makes evident that there must be a more systematic effort to identify and quantify the abilities of students. Diagnostic assessments will provide teachers and professors with information that will identify the sources of students' low performance.

For the Universidad Panamericana, both designing proper courses for reinforcement of the arithmetic and algebraic thinking of the students are important. The Universidad Panamericana also tries to transmit these results to high school directors and teachers, making emphasis on the importance to detect misconceptions when they appear in school mathematic lessons. The investigations show students' thinking to teachers, and may help to design school activities and confrontations with the errors.

\section{References}

Eccius, C. (2008). Mathematikdidaktische Fehleranalysen zur Schulalgebra: Schülerwissen und Lehrerprofessionswissen. Saarbrücken, Deutschland: VDM Verlag Dr. Müller.

Kieran, C. (1979). Children's operational thinking within the context of bracketing and the order of operations. In D. Tall (Ed.), Proceedings of the third international conference for The Psychology of Mathematics Education (pp. 128-135). Coventry, UK: Warwick University, Mathematics Education Research Centre.

Liebenberg, R. (1997). The usefulness of an intensive diagnostic test. In P. Kelsall, \& M. de Villiers (Eds.), Proceedings of The Third National Congress of the Association for Mathematics Education of South Africa, 2, 72-79. Durban: Natal University.

Malle, G. (1993). Didaktische Problema der elementaren Algebra. Wiesbaden, Deutschland: Vieweg.

Nolte, M. (1991). Strukturmomente des Unterrichts und ihre Bedeutung für das Lernen. Bad Salzdetfurth, Deutschland: Franzbecker

Padberg, F. (1996). Didaktik der Arithmetik (2nd ed.). Heidelberg, Deutschland: Spektrum Academischer Verlag GmbH.

Padberg, F. (2005). Didaktik der Arithmetik für Lehrerausbildung und Lehrerfortbildung (4th ed.). München, Deutschland: Spektrum, Elsevier GmbH.

Radatz, H. (1979). Error analysis in mathematics education. Journal for Research in Mathematics Education, 10, $163-172$.

Tietze, U. P., Klika, M., \& Wolpers, H. (Eds.). (2000). Mathematikunterricht in der Sekudarstufe II, Band 1 (2nd ed.). Germany: Vieweg.

Tirosh, D., Even, R., \& Robinson, N. (1998). Simplifying algebraic expressions: Teacher awareness and teaching approaches. Educational Studies in Mathematics, 35, 51-64. 
Vlassis, J. (2002). About the flexibility of the minus signs in solving equations. In A. D. Cockburn, \& E. Nardi (Eds.), Proceedings of The 26th Conference of the International Group of the Psychology of Mathematics Education, 4, 321-328. Norwich, UK: University of East Anglia.

Warren, E. (2003). The role of arithmetic structure in the transition from arithmetic to algebra. Mathematics Education Research Journal, 15, 122-137.

\section{Appendix}

Administrated Test

(1) Calculate, without calculator:

1. $25-5 \cdot 2+4=$

2. $2+3 \cdot \sqrt{3}-4=$

3. $9 \cdot 3 \div 6 \cdot 0=$

4. $-8^{2}=$

5. $\frac{8}{7} \cdot \frac{14}{2} \cdot \frac{9}{4}=$

6. $\frac{21}{45} \div \frac{7}{15}=$

7. $\sqrt{0.16}=$

8. $\sqrt{\frac{1}{9}}=$

9. $\sqrt[3]{-64}=$

10. $\sqrt{4^{2}+3^{2}}=$

(2) Simplify the algebraic expressions:

11. $10^{-7} \cdot \frac{10^{4}}{10^{-2}}=$

12. $b^{3 x+1} b^{1-3 x}=$

13. $\frac{(x-5)^{m+2}}{(x-5)^{m}}=$

14. $\frac{x^{\frac{3}{2}}}{x^{\frac{2}{5}}}=$

15. $\left(-3 x^{-4}\right)^{2}=$

16. $\sqrt[3]{\sqrt[6]{y^{36}}}=$

17. $\frac{\sqrt[3]{z^{4}}}{\sqrt[3]{z}}=$

18. $\sqrt{x^{2}+y^{2}}=$

(3) Make the operations and simplify:

19. $\frac{25 x^{-1} y^{4} z^{-2}}{-5 x^{2} y^{-1} z^{-4}}=$

20. $14 x^{2}-6 x y+8 x y-3 y^{2}=$ 
21. $3 \cdot(x-2)+4 \cdot(3-x)-(x-5)=$

22. $\left(z^{2}-5 z+4\right) \cdot(z-1)=$

23. $\sqrt{x^{2}+y^{2}}=$

(4) Factorize completely:

24. $z^{2}-16 z+64=$

25. $9 y^{2}+6 y+1=$

26. $x^{2}+1+3 x\left(x^{2}+1\right)=$

27. $x^{2}-12 x+35=$

28. $y^{3}-9 y=$

(5) Simplify the algebraic fractions:

29. $\frac{(x-5)(x+3)}{x+3}=$

30. $\frac{y \cdot(y+2)+(y+2)}{y+2}=$

31. $\frac{(z+4) \cdot(z-1)+2}{z+4}=$

32. $\frac{3}{a+b}-\frac{2}{a-b}=$

(6) Solve the following equations:

33. $x \cdot(3-x)-4 \cdot(1+x)+x \cdot(x-3)=0$

34. $6-\frac{2 x}{3}=9$

35. $\frac{x+3}{x-4}=0$

(7) Solve the following quadratic equations:

a) via factorization:

36. $x^{2}-4 x+3=0$

37. $2 x^{2}+2 x=0$

b) via general formula:

38. $2 x^{2}-x-15=0$

39. $z^{2}-9=0$

(8) Calculate the intersection of the lines:

40. $x-y=2$

$y-2 x=1$

(9) Graph the function:

41. $y=\frac{1}{2} x+1$

(10) 42. An article costs $\$ 232$, with $16 \%$ taxes, included. Which is the price before taxes? 